\title{
Testing dark matter distributions by neutrino-dark matter interactions
}

\author{
A. V. Penacchioni ${ }^{1,2, a}$, O. Civitarese ${ }^{1,2, b}$, C. R. Argüelles $^{3, c}$ \\ ${ }^{1}$ IFLP (CONICET), 1900 La Plata, Argentina \\ ${ }^{2}$ Dept. of Physics, University of La Plata, c.c. 67, 1900 La Plata, Argentina \\ ${ }^{3}$ Facultad de Ciencias Astronómicas y Geofísicas, Universidad Nacional de La Plata, Paseo del Bosque, B1900FWA La Plata, Argentina
}

Received: 18 October 2019 / Accepted: 12 February 2020 / Published online: 28 February 2020

(C) The Author(s) 2020

\begin{abstract}
At present, a variety of dark matter (DM) density profiles are available in the literature, able to fit the observed rotation velocity curves in galaxies. These distributions may be classified according to nature and mass of the DM candidate, and their estimation of the concentration of DM on halo scales, as well as through their central regions. Examples of these distributions are the (empiric) Einasto or isotropic, the (N-body-simulation-based) Navarro-Frenk-White (NFW), or the (elementary-particle-based) Ruffini-Argüelles-Rueda (RAR), among others. In this work, we calculate the crosssections for the interaction between neutrinos coming from astrophysical sources and DM particles subject to some of these distributions. Furthermore, we explore the changes in the neutrino oscillation patterns due to the different DM profiles used to represent the environment. From the results of the calculations it is concluded that both the cross-section and the neutrino oscillation pattern depend on the features of each of the adopted models for the DM distributions. For the case of the NFW and isotropic DM distributions the expected effects at Earth, for neutrinos coming from an extragalactic source, are noticeable while in the case of the RAR distribution the effects are restricted to a narrow region around the galactic center.
\end{abstract}

\section{Introduction}

One of the most intriguing aspects in modern astrophysics and cosmology is the presence of dark matter (DM) in the Universe. The name encompasses all possible forms of matter which is not detectable by the radiation it may emit. Its presence is inferred through gravitational effects like the ones

\footnotetext{
a e-mail: ana.penacchioni@ fisica.unlp.edu.ar (corresponding author)

b e-mail: osvaldo.civitarese@ fisica.unlp.edu.ar

c e-mail: charly@carina.fcaglp.unlp.edu.ar
}

observed in the Cosmic Microwave Background (CMB), changes in the rotation velocity of galaxies, and departures from the expected values of the parameters measured in the expansion of the Universe [1].

The properties and composition of DM are unknown, although the most favored candidates for cold DM are neutral leptons [1,2]. The non-photonic interactions of these leptons with other particles can be described at tree-level by Lagrangians where the mediators are bosons. They can be also treated by Lagrangians which contain operators of order five or larger [3]. In addition to the unknown properties of DM particles, their space distribution becomes a central issue. One may think of various scenarios where the concentration of DM may be different in the central and the outer region of the galaxies, and in the intergalactic space. During the last decades, a great effort has been invested to determine an acceptable morphology for DM distributions considering the observation of the rotation curves (see e.g. [4-6]). Among these distributions one can mention the (N-bodysimulation-based) Navarro-Frenk-White (NFW) [7-9], the (empiric) Einasto [10], the isotropic [11], the elementaryparticle based RAR [12-14], etc. We shall discuss the features of some of these distributions in the next Section. In fitting these distributions to the observations also the mass $m_{D M}$ of the DM particles plays an important role. Indeed, the DM halo structures arising either within the cold (for particle masses between a few and a hundred $\mathrm{GeV}$ [15]) or warm (for particles in the $\mathrm{keV}$ regime) DM paradigms are well explained in terms of the NFW profile [16]. In the case of the RAR model the range of masses for DM particles, in agreement with core-halo Galactic observables, varies from 10 to $10^{2} \mathrm{keV}[12,14]$.

Neutrinos are an essential part of practically all astrophysical processes. They are neutral, spin-1/2 fermions which in the Standard Model (SM) are massless [17]. They come in three-flavors. The existence of neutrino oscillations between 
different flavors indicates that they are linear superpositions of three eigenstates of a mass Hamiltonian, $H_{m}$ [18]. The absolute values of these three mass eigenstates are unknown, but the squared differences between them can be determined experimentally. From the measurements of flavoroscillations for atmospheric and solar neutrinos, the mixing angles and squared mass differences are determined [1922]. Neutrinos can interact with other particles via neutral and charged currents by exchanging bosons $\left(W^{ \pm}, Z^{0}\right)$ of the SM or with other particles in models beyond the SM [3,23].

Since neutrinos can change their flavor composition while traveling from the source to the detector, it is then extremely appealing to think that neutrinos produced outside our galaxy can evolve on their way to Earth through interactions with DM particles. In previous works [24-26] we have explored this possibility and found that it is indeed the case.

As we shall see in the next sections, the resulting amplitudes describing the scattering of neutrinos by DM particles are explicitly dependent on the mass of the latter, and the cross sections include the dependence on the DM space distribution through changes in the elements of the neutrino mixing matrix. The aim of this paper is to compare the results for the cross sections for the neutrino-DM scattering for different values of the mass of the DM particles and their distributions. In the next section we shall explicitly define the DM distributions, the vertices for the neutrino-DM scattering and the corresponding probabilities.

The paper is organised as follows: in Sect. 2.1 we introduce the DM distributions; in Sect. 2.2 the neutrino-DM interactions and the corresponding cross sections. In Sect. 3 we present and discuss the results of our calculations. Finally, in Sect. 4 we draw our conclusions.

\section{Formalism}

\subsection{DM distributions}

Starting from Milky Way rotation curve data, as provided for example in [9], it is possible to write down matter distributions for DM particles taking into account certain model assumptions. In the case of the NFW distribution [7,8], DM is concentrated around the GC and dies out according to the expression:

$\rho_{\mathrm{NFW}}(r)=\rho_{\oplus}\left(\frac{r_{\oplus}}{r}\right)\left(\frac{1+\left(r_{\oplus} / r_{s}\right)}{1+\left(r / r_{s}\right)}\right)^{2}$,

where $r_{s}=20 \mathrm{kpc}, \rho_{\oplus}=0.4 \mathrm{GeV} \mathrm{cm}^{-3}$ is the local DM density and $r_{\oplus}=8.5 \mathrm{kpc}$ is the distance from the Solar System to the $\mathrm{GC}$, in agreement with the phenomenological work by [27]. Another parameterized form of the DM distribution is the isotropic one:

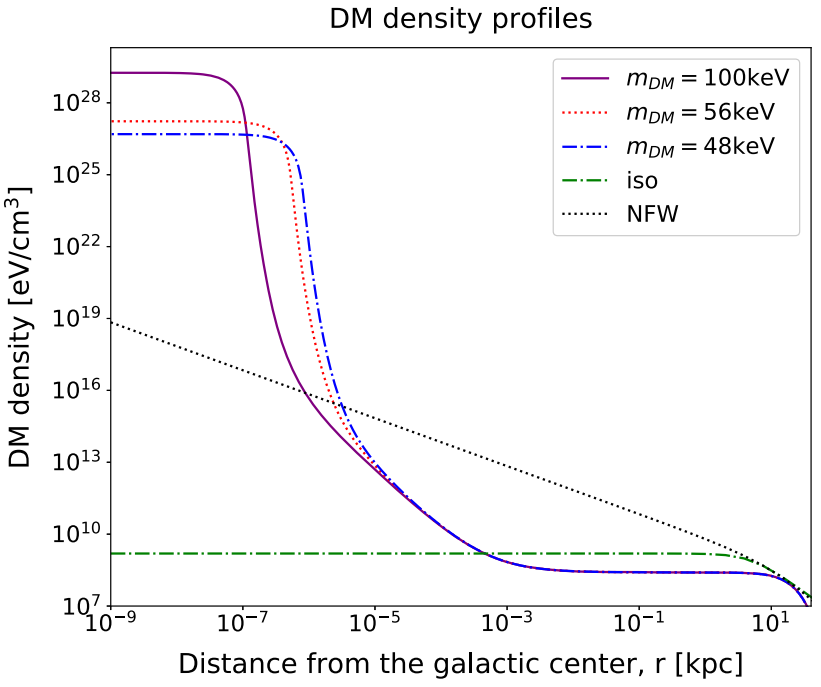

Fig. 1 DM density profiles as a function of the radial distance from the GC. The distributions considered are isotropic (green dashed-dot line, see Eq. 2), Navarro-Frenk-White (black dotted line, see Eq. 1), RAR for $m_{D M}=100 \mathrm{keV}$ (solid line), $m_{D M}=56 \mathrm{keV}$ (red dotted line) and $m_{D M}=48 \mathrm{keV}$ (blue dashed line). We notice that all the distributions are very similar around $10 \mathrm{kpc}$, where most of the rotation curve data is available

$\rho_{\text {iso }}(r)=\rho_{\oplus}\left(\frac{1+\left(r_{\oplus} / r_{s}\right)^{2}}{1+\left(r / r_{s}\right)^{2}}\right)$

with $r_{s}=5 \mathrm{kpc}$ as inferred in [28]. This distribution has a finite value around the GC (i.e. develops a constant density plateau) and decreases as $r^{-2}$ at large distances from the GC. A third form of DM distribution is the RAR distribution [1214], described in terms of a self-gravitating system of elementary neutral keV-fermions including Pauli principle effects through the central region and the escape of particles in the outer tail. These profiles imply a continuous distribution of DM, with a very dense and compact core governed by Fermi degeneracy and confined within mpc scales, followed by a core-halo transition towards a Boltzmannian behaviour (i.e. thermal pressure supported and bounded in radius). Another difference between these distributions is that in the case of RAR the mass of DM particles affects the compactness of the central core, as in the Milky Way fit of Refs. [12,14]. Figure 1 shows the radial dependence of different RAR distributions which best fit the Milky Way rotation curve [12], together with the radial dependence corresponding to the NFW and Isotropic DM distributions [28].

\subsection{Neutrino-DM interactions}

Figure 2 illustrates the exchange of a mediator between the currents of neutrinos and DM particles. The resulting amplitude is written 
$\mathcal{M}=\partial_{\nu \nu}^{\alpha}\left(\frac{g_{\alpha \beta}+k_{\alpha} k_{\beta} / M_{Z^{\prime}}^{2}}{k^{2}-M_{Z^{\prime}}^{2}}\right) \partial_{X X}^{\beta}$,

where

$\mathcal{J}_{v \nu}^{\alpha}=\bar{v}\left(p^{\prime}, s^{\prime}\right) \gamma^{\alpha} \frac{\left(1-\gamma^{5}\right)}{2} v(p, s)$

is the current of the neutrino sector and

$\partial_{X X}^{\beta}=\bar{X}\left(q^{\prime}, r^{\prime}\right) \gamma^{\beta} \frac{\left(1-\gamma^{5}\right)}{2} X(q, r)$

is the DM current. The term between parenthesis in Eq. (3) is the propagator of the exchanged boson. In Eqs. (4) and (5), $v(p, s)\left(\bar{v}\left(p^{\prime}, s^{\prime}\right)\right)$ are neutrino Dirac spinors, with incoming (outgoing) momentum $p\left(p^{\prime}\right)$ and spin $s\left(s^{\prime}\right) . X(q, r)$ $\left(\bar{X}\left(q^{\prime}, r^{\prime}\right)\right)$ are Dirac spinors for DM particles with incoming (outgoing) momentum $q\left(q^{\prime}\right)$ and $\operatorname{spin} r\left(r^{\prime}\right)$.

In the limit $M_{Z^{\prime}}>>k$, Eq. (3) reduces to

$\mathcal{M}=\mathcal{J}_{\nu \nu}^{\alpha}\left(\frac{g_{\alpha \beta}}{M_{Z^{\prime}}^{2}}\right) \partial_{X X}^{\beta}$.

Since neutrinos of a given flavor $|v\rangle_{f}$ are linear superpositions of neutrino-mass eigenstates

$|v\rangle_{f}=\sum_{k} U_{f k}\left|m_{k}\right\rangle$,

where $U_{f k}$ are the elements of the mixing matrix and where $\mathrm{CP}$-violation phases are ignored, the vertex in Fig. 2 should be decomposed into the corresponding interactions between neutrino-mass eigenstates.

To calculate the amplitude $\mathcal{M}$ of Eq. (3), corresponding to the diagram in Fig. 2, we expand the neutrino and DM currents using Dirac spinors, making use of completeness relations and taking traces over spin variables. The algebraic procedure, although a little demanding, is straightforward. The interaction probability is given by squaring $\mathcal{M}$, summing over the spin projections and integrating in 4-space for a given combination of initial and final neutrino-mass eigenstates. The final result is given by

$$
\begin{aligned}
\sum_{\text {spins }}|\mathcal{M}|^{2}= & \frac{8 g_{w}^{2} \pi^{4}\left(N_{p} N_{p^{\prime}} N_{q} N_{q^{\prime}}\right)^{2}}{2(\hbar c)^{2} M_{Z^{\prime}}^{4} \lambda^{4}} V T \delta^{(4)} \\
& \left(p+p^{\prime}+q+q^{\prime}\right) \\
\times & {\left[2\left(p^{\prime} \cdot q^{\prime}\right)(p \cdot q)+m_{D M}^{2}\left(p \cdot p^{\prime}\right)+m_{v_{i}} m_{v_{f}}\left(q \cdot q^{\prime}\right)\right.} \\
& \left.+2 m_{D M}^{2} m_{v_{i}} m_{v_{f}}\right]
\end{aligned}
$$

where $m_{v_{i}}\left(m_{v_{f}}\right)$ is the mass of the incoming (outgoing) neutrino, $m_{D M}$ is the mass of the DM particles, $N_{k}\left(k=p, p^{\prime}\right.$, $q, q^{\prime}$ ) is the norm of the spinor of the neutrino (DM particle) with momentum $k, \lambda$ is the coupling constant and $g_{w}$ is the strength of the interaction of the neutrinos and DM particles with the mediator boson $\left(Z^{\prime}\right) . V$ is the volume in which the interaction takes place and $T$ is the time. They appear when using the delta property $\left[\delta^{(4)}\left(p+p^{\prime}+q+q^{\prime}\right)\right]^{2}=$ $\delta^{(4)}\left(p+p^{\prime}+q+q^{\prime}\right) \delta^{(4)}(0)=\frac{V T}{(2 \pi)^{4}} \delta^{(4)}\left(p+p^{\prime}+q+q^{\prime}\right)$. Expression 8 is valid for any pair of neutrino-mass eigenstates with masses $m_{v_{i}}$ and $m_{v_{f}}$ and four-momenta $p$ and $p^{\prime}$, and for DM particles with four-momenta $q$ and $q^{\prime}$ and mass $m_{D M}{ }^{1}$

The differential cross section $\frac{d \sigma}{d \Omega}$ for the scattering of neutrinos by DM particles is given by the expression

$\frac{d \sigma}{d \Omega}=\frac{|\mathcal{M}|^{2}}{64 \pi^{2} E_{\text {scale }}^{2}}$,

with $E_{\text {scale }}=50676.5317[1 / \mathrm{cm}]$. This scale factor comes out from considering natural units $(\hbar c=1)$. Integrating in angles we obtain

$\sigma(p)=\int \frac{|\mathcal{M}|^{2}}{64 \pi^{2} E_{\text {scale }}^{2}} d \Omega$

To account for the composite nature of the neutrinos, the cross section (10) should include the elements of the mixing matrix $U$ for the initial and final neutrino states, and the summation over all mass-eigenstates, as dictated by Eq. (7). The transformation from the center of momentum (CM) to the laboratory frame is performed by applying the rules of special relativity.

In actual calculations we have adopted the squared-mass differences extracted from the available neutrino oscillation data [29] and $m_{D M}$ is varied according to each model for the DM distribution. Concerning the values of $U$, they will reflect the local dependence given by the DM distribution, as explained in the next section (see also [24,25]).

\section{Results}

In this section we shall present and discuss the results of our calculations, which have been performed by considering electron-neutrinos emitted from an extragalactic source, located at a distance $l$ from Earth. These neutrinos have four-momentum $p$, and are linear combinations of mass-eigenstates that obey the Normal Hierarchy $(\mathrm{NH})$ with squared-mass differences and mixing amplitudes taken from the latest compilation of PDG [29]. To this initial condition we add the details of DM distributions and DM masses, and

\footnotetext{
${ }^{1}$ In expression 8 the dot indicates the Lorentz product between fourmomenta.
} 


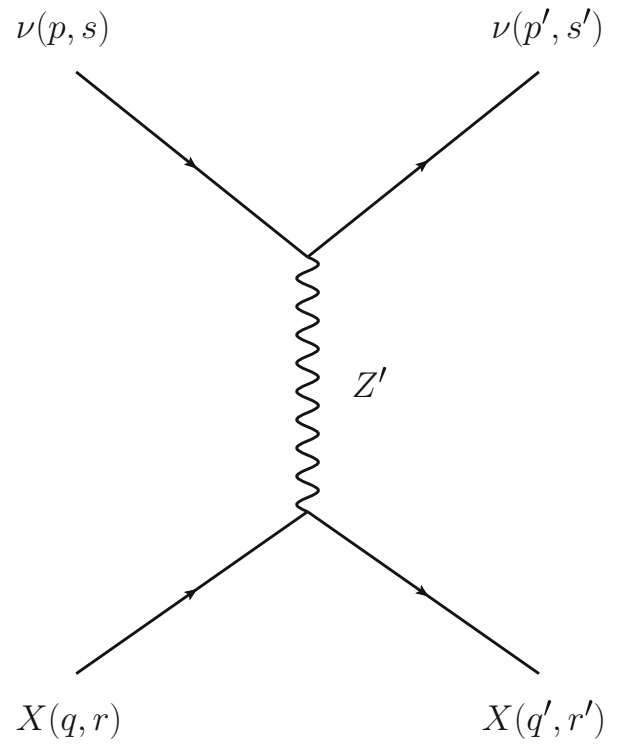

Fig. 2 Feynman diagram representing the interaction between electron neutrinos $(v)$ and DM particles $(X)$ through a $Z^{\prime}$ boson mediator

fix the trajectories of the neutrinos so that they pass through the GC, as explained in Penacchioni and Civitarese [24].

\subsection{Main features of the DM distributions}

Figure 1 shows the different DM distributions currently adopted to fit the rotation curves of the Galaxy. The differences among them are noticeable at distances from the GC that vary from $r \approx 0$ to $r=10 \mathrm{kpc}$. In the NFW case, the density grows up as an inverse power of $\mathrm{r}\left(\propto r^{-1}\right)$ towards the GC and starts to vanish as $r^{-3}$ for distances $r>r_{s}$. The isotropic distribution, on the contrary, exhibits a finite value at the $\mathrm{GC}$, which is several orders of magnitude smaller than the maximum values of the rest of the distributions. At variance from these distributions, the RAR ones display a stepfunction-like dependence [12]. It is confined to very small distances (below mpc scales) around the GC, and vanishes for $r \sim 40 \mathrm{kpc}$. The values of $m_{D M}$ enter explicitly in the parameterization of the RAR distributions: the larger $m_{D M}$ the more compact the central core and the higher the value of $\rho_{0}$.

The set of parameters governing the pattern of neutrino oscillations changes if neutrinos interact with matter. This is known as the MSW effect [30-32]. The interaction of neutrinos with the environment may also produce the onset of decoherence, as pointed out in recent works [24, 28,33]. In dealing with these interactions, when the environment is composed by DM particles, there are several unknown parameters to be determined, like the coupling constants of the Lagrangian describing the interactions, the mass of the DM particles and their distribution, the texture of the interaction matrix $\Lambda$ [24], etc. For the purpose of the present calculations we shall esti- mate the change in the neutrino-oscillation pattern due to interactions with DM. The formalism has been presented in Penacchioni and Civitarese [25,26]. For the sake of concreteness we shall omit giving the details here. Briefly speaking, the procedure consists of successive diagonalizations of the Hamiltonian $H_{f}+\lambda V(r)$, where $\lambda$ is a renormalization constant, $H_{f}$ is the flavor Hamiltonian and $V(r)$ is the locally defined DM-depending interaction [28,33], which takes the form

$V=G_{F} \frac{\rho(r)}{m_{D M}} \Lambda$.

Here, $G_{F}=8.963 \times 10^{-44} \mathrm{MeV} \mathrm{cm}^{3}$ is the Fermi constant, $\rho(r)$ is the DM density distribution, $m_{D M}$ is the DM mass in units of energy, and $\Lambda$ is a $3 \times 3$ matrix (called the texture matrix) through which we can vary the degree of neutrino mixing, from single scaling to decoherence.

From the diagonalization of such a Hamiltonian the amplitudes of the mixing matrix are extracted at different points along the neutrino path.

As examples, we are listing below the values of $U$ for $\rho_{N F W}(r)$ and $\rho_{i s o}(r)$, taking $m_{D M}=100 \mathrm{keV}$ and for neutrino trajectories passing through the $\mathrm{GC}$, and for a distance of $20 \mathrm{kpc}$ between the source and the detector ${ }^{2}$ [24]. The chosen values of the distance between the interaction point and the GC are: at the source $(r=11.5 \mathrm{kpc}, t=0)$, near the $\mathrm{GC}\left(r=0.1 \mathrm{kpc}, t=1.1969940037 \times 10^{12} \mathrm{~s}\right)$ and at the detector on Earth, $\left(r=8.5 \mathrm{kpc}, t=2.063782765 \times 10^{12} \mathrm{~s}\right)$.

For $\rho_{i s o}(r)$, the mixing matrix at these values of $r$ are:

$$
\begin{gathered}
U_{\text {source }}=\left[\begin{array}{ccc}
0.8293 & 0.5390 & 0.1466 \\
-0.4933 & 0.5836 & 0.6448 \\
0.2620 & -0.6071 & 0.7500
\end{array}\right], \\
U_{\text {near } G C}=\left[\begin{array}{ccc}
0.8052 & 0.5798 & -0.1240 \\
-0.5083 & 0.5673 & -0.6478 \\
-0.3052 & 0.5847 & 0.7516
\end{array}\right], \\
U_{\text {detector }}=\left[\begin{array}{ccc}
0.8560 & 0.4875 & -0.1715 \\
-0.4731 & 0.6058 & -0.6395 \\
-0.2079 & 0.6287 & 0.7493
\end{array}\right] .
\end{gathered}
$$

For $\rho_{N F W}(r)$, these values are:

$$
\begin{gathered}
U_{\text {source }}=\left[\begin{array}{ccc}
0.8293 & 0.5390 & 0.1466 \\
-0.4933 & 0.5836 & 0.6448 \\
0.2620 & -0.6071 & 0.7500
\end{array}\right], \\
U_{\text {near } G C}=\left[\begin{array}{ccc}
-0.0708 & 0.9974 & -0.0019 \\
0.7157 & 0.0494 & -0.6965 \\
0.6947 & 0.0507 & 0.7174
\end{array}\right],
\end{gathered}
$$

\footnotetext{
${ }^{2}$ Neutrino trajectories $(r)$ are parameterized by the distance between the detector on Earth and the source $(l)$, the position of the GC with respect to the Earth $\left(r_{\oplus}\right)$ and by the angle $\phi$ between them, as shown in Figure 1 of [24]. The examples given in the text correspond to $\phi=0$.
} 
$U_{\text {detector }}=\left[\begin{array}{ccc}0.8371 & 0.4040 & -0.3687 \\ -0.4883 & 0.8556 & -0.1711 \\ 0.2463 & 0.3233 & 0.9136\end{array}\right]$.

We repeat our calculations for $\rho_{R A R}(r)$ for the same values of the parameters. For this choice, the $U$ matrix does not change with distance and keeps the initial values (those of Eq. 12) all along the neutrino trajectory, except for extremely small values of $r$ (see Fig. 1).

\subsection{Results for the cross section $\sigma_{v-D M}$}

As a first step, we have calculated the cross-section for $v-D M$ interactions, taking different values of $m_{D M}$ and varying the momentum of the incoming neutrino. The curves shown in Fig. 3 represent the integral over angles of the differential cross section of Eq. (9), for different DM masses. These values are independent of the DM distribution, i.e. independent of the location where the scattering takes place. In this example, the values chosen for the DM masses are those of the RAR distribution. The incoming (outgoing) neutrinos have an average mass $m_{v_{i}}\left(m_{v_{f}}\right)=0.1 \mathrm{eV}$.

To give an idea of the local effect upon the cross section induced by the flux of DM particles at a fixed point in space, we list here three values of the integrated cross section at a position $r$. These values have been obtained after computing the amplitudes $U$ for an effective potential $V(r) \propto \frac{\rho_{i s o}(r)}{m_{D M}} \Lambda_{\text {diag }}(1,1,1), \lambda=10^{15}$ and for $r=$ $11.5 \mathrm{kpc}$ (source), $r=0.1 \mathrm{kpc}$ (near the GC) and $r=8.5 \mathrm{kpc}$ (detector), for $p=50 \mathrm{keV}$ :

$\sigma(p, r=11.5 \mathrm{kpc})=2.24 \times 10^{-33} \mathrm{~cm}^{2}$,

$\sigma(p, r=0.1 \mathrm{kpc})=1.55 \times 10^{-35} \mathrm{~cm}^{2}$ and

$\sigma(p, r=8.5 \mathrm{kpc})=6.24 \times 10^{-35} \mathrm{~cm}^{2}$, respectively. These values have been obtained by setting $g_{w}=1$. The curves representing the calculated cross-section for different values of $p$ are shown in Fig. 4. These values can be compared with the one obtained for structure-less neutrinos with average mass of $0.1 \mathrm{eV}$ and momentum $p=50 \mathrm{keV}(\sigma=4.26 \times$ $10^{-34} \mathrm{~cm}^{2}$ ), which is the same value calculated for the RAR distribution. Obviously, the choice of a particular texture for $\Lambda$ is arbitrary. A relatively systematic study of the possible configurations or textures is reported in [28], for a wide range of values of the DM mass and the renormalization coupling constant $\lambda$. Since in this context we are interested in exploring the gross effect upon the neutrino oscillations caused by local interactions with DM, we have taken a value of $\Lambda$ which mostly affects the neutrino mixing, that is the diagonal form.

\subsection{Neutrino oscillations in presence of DM}

Another indication of the presence of DM in the space between extragalactic sources and the Earth could be the change in the pattern of neutrino oscillations. As discussed

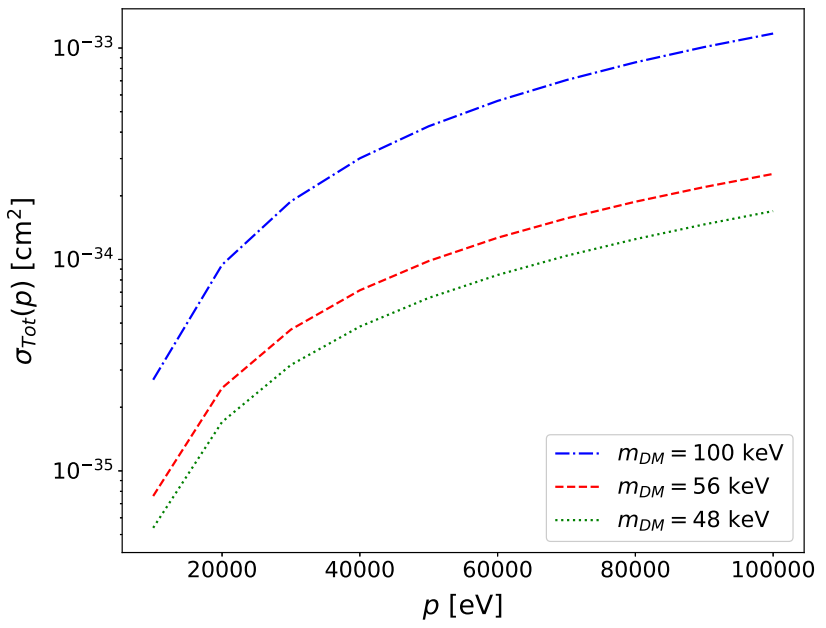

Fig. 3 Cross section integrated in solid angle as a function of the neutrino initial momentum $p$ for different values of $m_{D M}$ (see Eq. 8)

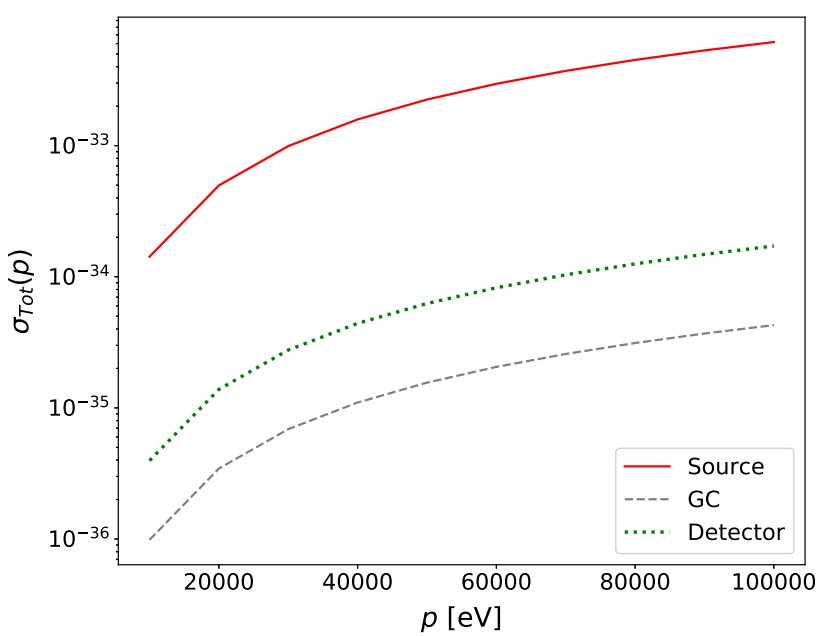

Fig. 4 Cross section integrated in solid angle as a function of the neutrino initial momentum $p$ for $m_{D M}=100 \mathrm{keV}$, including the mixing between neutrino mass-eigenstates in the electron neutrino $v_{e}$. These results have been obtained by using $\rho_{i s o}(r)$, for different values of $r$. The corresponding positions are denoted by source $(r=11.5 \mathrm{kpc}), \mathrm{GC}$ $(r=0.1 \mathrm{kpc})$ and Detector $(r=8.5 \mathrm{kpc})$

in de Salas et al. [28], Penacchioni and Civitarese [24] and Choi et al. [33], changes in the pattern of oscillations due to localized DM density distributions are expected. Like in the MSW mechanism, where the oscillations are modified by normal matter, interactions with the potential $V(r)$ induce changes in the elements of the mixing matrix $U$ that affect the survival $P_{v_{f} \rightarrow v_{f}}$ and disappearance $P_{v_{f} \rightarrow v_{f}^{\prime}}$ probabilities for neutrinos of a given flavor. Figure 5 shows the results of our calculations for the survival and disappearance probabilities for electron neutrinos produced at a distant source located at $20 \mathrm{kpc}$ away from Earth. The parameters and models used in the calculations are specified in the caption to the figure. It can be seen that the 


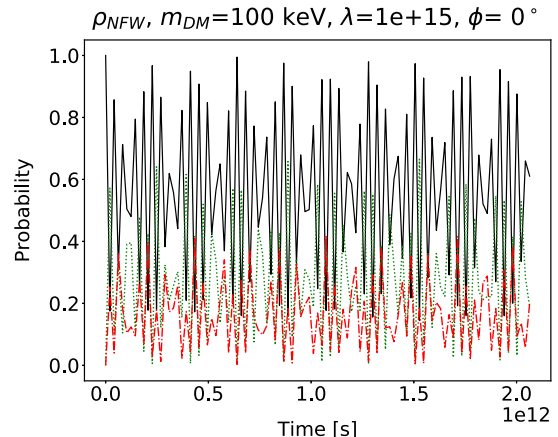

(a)

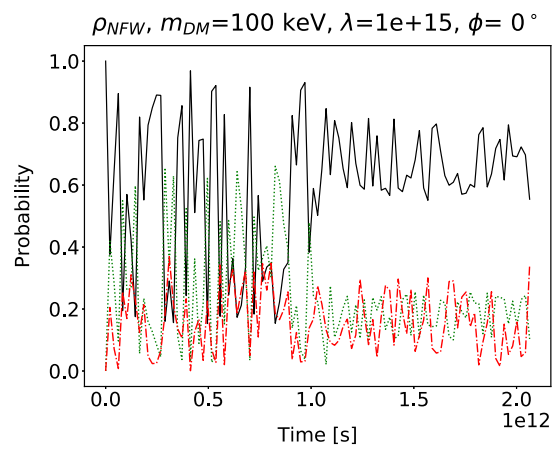

(d)

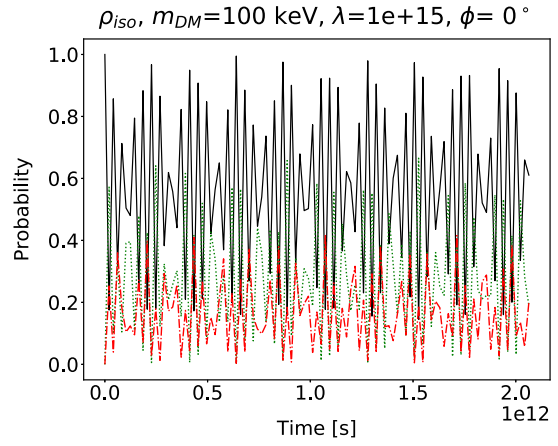

(b)

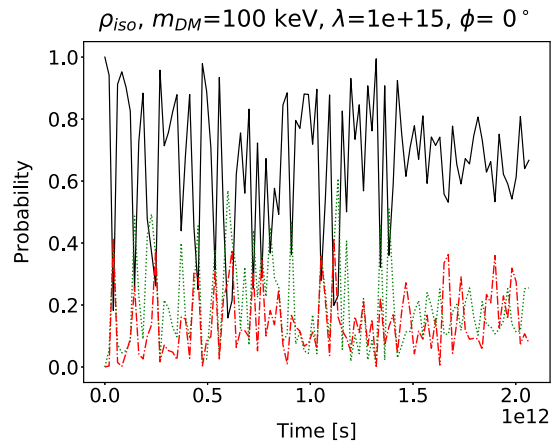

(e)

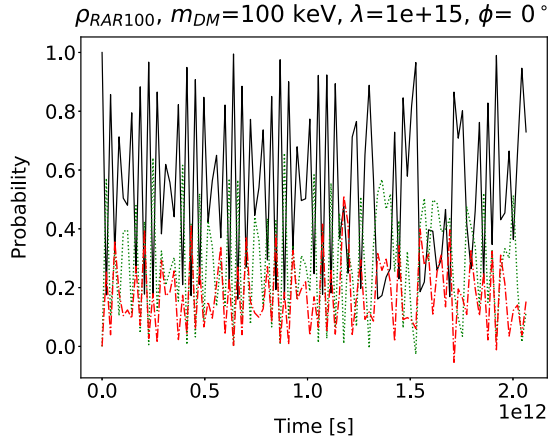

(c)

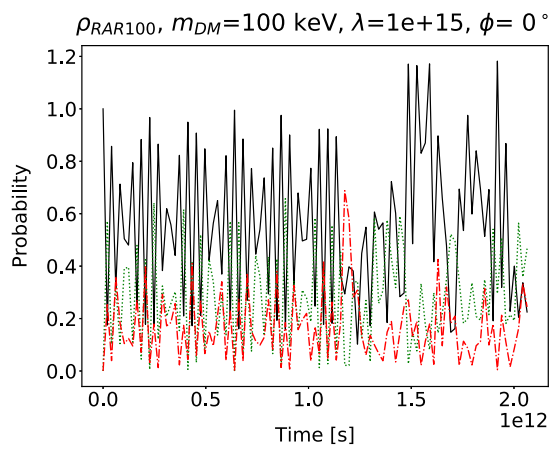

(f)
Fig. 5 Survival and disappearance probabilities in presence of DM as a function of time, for a value of the DM mass $m_{D M}=100 \mathrm{keV}$ and for the NFW (a, d), isotropic (b, e) and RAR (c, f) DM density profiles, considering different textures for the matrix $\Lambda$ (cases $\mathbf{a}-\mathbf{c}$ correspond to $\Lambda=I_{3 \times 3}$, and cases $\mathbf{d}-\mathbf{f}$ correspond to $\Lambda_{11}=\Lambda_{33}=-1, \Lambda_{i k}=0$

changes induced by the RAR distribution are confined to a very narrow region around the GC. For the NFW and the isotropic DM distributions the onset of decoherence effects becomes evident for neutrinos once they have passed across the GC.

If we combine these results with those of the previous Section, we may conclude that the RAR distribution, although useful in the fit of the rotation velocity curves, does not affect much neutrino properties except at the very GC.

In order to illustrate better the effects of the neutrinoDM interactions upon the pattern of neutrino oscillations, we calculated the averaged survival/transition probabilities for each neutrino flavor $\left(\overline{P_{v_{e} \rightarrow v_{e}}}, \overline{P_{v_{e} \rightarrow v_{\mu}}}\right.$ and $\left.\overline{P_{v_{e} \rightarrow v_{\tau}}}\right)$ in vacuum and for each case of Fig. 5. The values are shown in Table 1. From the results shown in the table it is seen that the average neutrino survival probabilities of a given flavor remain the same vacuum values for cases labelled (a), (b) and (c), which correspond to single scaling. On the contrary, changes are significant for the other cases of non-diagonal interactions thus signalling the influence of decoherence. otherwise). The solid line represents the electron flavor, the dotted line the $\mu$ flavor and the dashed line the $\tau$ flavor. We assume that neutrinos are emitted at the source with energy $E_{v}=1 \mathrm{TeV}$ and their trajectory passes through the GC $(\phi=0)$

Table 1 Average values of the survival/transition probabilities for each neutrino flavor in vacuum $(\lambda=0)$ and for the set of parameters corresponding to each case of Fig. 5. For cases $5 \mathrm{a}$ and $5 \mathrm{~b}$ the values differ from those in vacuum only in the fifth decimal place

\begin{tabular}{llll}
\hline Case & $\overline{P_{v_{e} \rightarrow v_{e}}}$ & $\overline{P_{v_{e} \rightarrow v_{\mu}}}$ & $\overline{P_{v_{e} \rightarrow v_{\tau}}}$ \\
\hline Vacuum & 0.56 & 0.27 & 0.16 \\
Figure 5a & 0.56 & 0.27 & 0.16 \\
Figure 5b & 0.56 & 0.27 & 0.16 \\
Figure 5c & 0.56 & 0.26 & 0.16 \\
Figure 5d & 0.60 & 0.24 & 0.15 \\
Figure 5e & 0.65 & 0.19 & 0.14 \\
Figure 5f & 0.55 & 0.28 & 0.16 \\
\hline
\end{tabular}

\section{Conclusions}

In this work we have calculated the cross sections for $v$-DM scattering as a function of the DM density and masses, for different values of the neutrino momentum. To complement the information coming from the fit of the velocity curves we have explored the consequences of another criterion: the 
changes in the neutrino cross section and oscillation pattern due to the presence of DM. To this extent and following the line of thought of Refs. [24,28,33] we have performed a comparison of results for the NFW and isotropic distributions with the RAR ones. As explained in the text, the RAR distribution seems to affect extragalactic neutrinos only once they pass through the GC, making these effects difficult to observe at Earth, though it may imply an important and independent venue to test the DM concentration around the GC. The sensitivity of extragalactic-neutrino observables (mean energies, cross sections, oscillation patterns, etc) upon $\rho_{D M}(r)$ may indeed be a good test for DM models, constraining the value of the parameters extracted from the velocity curves.

Acknowledgements This work has been partially supported by the National Research Council of Argentina (CONICET) by the grant PIP 616, and by the Agencia Nacional de Promoción Científica y Tecnológica (ANPCYT) PICT 140492. The authors are members of the Scientific Research career of the CONICET. This work is part of the PUE -IFLP (CONICET).

Data Availability Statement This manuscript has no associated data or the data will not be deposited. [Authors' comment: Data sharing not applicable to this article as no datasets were generated or analysed during the current study.]

Open Access This article is licensed under a Creative Commons Attribution 4.0 International License, which permits use, sharing, adaptation, distribution and reproduction in any medium or format, as long as you give appropriate credit to the original author(s) and the source, provide a link to the Creative Commons licence, and indicate if changes were made. The images or other third party material in this article are included in the article's Creative Commons licence, unless indicated otherwise in a credit line to the material. If material is not included in the article's Creative Commons licence and your intended use is not permitted by statutory regulation or exceeds the permitted use, you will need to obtain permission directly from the copyright holder. To view a copy of this licence, visit http://creativecomm ons.org/licenses/by/4.0/.

Funded by SCOAP ${ }^{3}$.

\section{References}

1. G.B. Gelmini, in Journeys Through the Precision Frontier: Amplitudes for Colliders (TASI 2014), ed. by L. Dixon, et al. (2016), pp. 559-616. https://doi.org/10.1142/9789814678766_0012

2. G.B. Gelmini (2011). arXiv:1106.6278 (arXiv e-prints )

3. A. Boyarsky, O. Ruchayskiy, M. Shaposhnikov, Annu. Rev. Nucl. Part. Sci. 59(1), 191 (2009). https://doi.org/10.1146/annurev.nucl. 010909.083654

4. W.J.G. de Blok, F. Walter, E. Brinks, C. Trachternach, S.H. Oh, R.C. Kennicutt Jr., Astron. J. 136, 2648 (2008). https://doi.org/10. 1088/0004-6256/136/6/2648

5. M.G. Walker, M. Mateo, E.W. Olszewski, J. Peñarrubia, N.W. Evans, G. Gilmore, Astrophys. J. 704, 1274 (2009). https://doi. org/10.1088/0004-637X/704/2/1274
6. F. Lelli, S.S. McGaugh, J.M. Schombert, Astron. J. 152, 157 (2016). https://doi.org/10.3847/0004-6256/152/6/157

7. J.F. Navarro, C.S. Frenk, S.D.M. White, Astrophys. J. 462, 563 (1996). https://doi.org/10.1086/177173

8. J.F. Navarro, C.S. Frenk, S.D.M. White, Astrophys. J. 490, 493 (1997). https://doi.org/10.1086/304888

9. Y. Sofue, PASJ 64, 75 (2012). https://doi.org/10.1093/pasj/64.4.75

10. J. Einasto, Trudy Astrofizicheskogo Inst. Alma-Ata 5, 87 (1965)

11. J. Binney, S. Tremaine, Galactic Dynamics, 2nd edn. (Princeton University Press, Princeton, 2008)

12. C.R. Argüelles, A. Krut, J.A. Rueda, R. Ruffini, Phys. Dark Univ. 21, 82 (2018). https://doi.org/10.1016/j.dark.2018.07.002

13. C.R. Argüelles, A. Krut, J.A. Rueda, R. Ruffini, Phys. Dark Univ. 24, 100278 (2019). https://doi.org/10.1016/j.dark.2019.100278

14. C.R. Argüelles, A. Krut, J.A. Rueda, R. Ruffini (2019). arXiv:1905.09776 (arXiv e-prints)

15. G. Bertone, Particle Dark Matter: Observations, Models and Searches (Cambridge University Press, Cambridge, 2010)

16. S. Bose, W.A. Hellwing, C.S. Frenk, A. Jenkins, M.R. Lovell, J.C. Helly, B. Li, V. Gonzalez-Perez, L. Gao, MNRAS 464, 4520 (2017). https://doi.org/10.1093/mnras/stw2686

17. E. Leader, E. Predazzi, An Introduction to Gauge Theories and Modern Particle Physics 2 Volume Set (Hardback) (Cambridge University Press, Cambridge, 1996)

18. S. Pascoli, S.T. Petcov, T. Schwetz, Nucl. Phys. B 734(1-2), 24 (2006). https://doi.org/10.1016/j.nuclphysb.2005.11.003

19. Y. Fukuda et al., Phys. Rev. Lett. 81(8), 1562 (1998). https://doi. org/10.1103/PhysRevLett.81.1562

20. Q.R. Ahmad et al., Phys. Rev. Lett. 87(7), 071301 (2001). https:// doi.org/10.1103/PhysRevLett.87.071301

21. K. Abe et al., Phys. Rev. D 83(5), 052010 (2011). https://doi.org/ 10.1103/PhysRevD.83.052010

22. Q.R. Ahmad et al., Phys. Rev. Lett. 89(1), 011301 (2002). https:// doi.org/10.1103/PhysRevLett.89.011301

23. J.W.F. Valle, American Institute of Physics Conference Series, vol. 359, pp. 42-146 (1996). https://doi.org/10.1063/1.49733

24. A.V. Penacchioni, O. Civitarese (2019). arXiv:1904.04355 (arXiv e-prints)

25. A.V. Penacchioni, O. Civitarese, Astrophys. J. 872, 73 (2019). https://doi.org/10.3847/1538-4357/aafe7b

26. A.V. Penacchioni, O. Civitarese, Astrophys. J. Lett. 871(2), L30 (2019). https://doi.org/10.3847/2041-8213/ab0087

27. M. Pato, F. Iocco, G. Bertone, J. Cosmol. Astropart. Phys. 12, 001 (2015). https://doi.org/10.1088/1475-7516/2015/12/001

28. P.F. de Salas, R.A. Lineros, M. Tórtola, Phys. Rev. D 94(12), 123001 (2016). https://doi.org/10.1103/PhysRevD.94.123001

29. M. Tanabashi et al., Phys. Rev. D 98, 030001 (2018). https://doi. org/10.1103/PhysRevD.98.030001

30. A.Y. Smirnov, Phys. Script. Vol. T 121, 57 (2005). https://doi.org/ 10.1088/0031-8949/2005/T121/008

31. L. Wolfenstein, Phys. Rev. D 17(9), 2369 (1978). https://doi.org/ 10.1103/PhysRevD.17.2369

32. S.P. Mikheyev, A.Y. Smirnov, Yad. Fiz. 42, 1441 (1985)

33. K.Y. Choi, E.J. Chun, J. Kim (2019) arXiv:1909.10478 (arXiv eprints) 